\title{
Synergistic Effect of Pasireotide and Teriflunomide in Carcinoids in vitro
}

\author{
Yash Somnay Herbert Chen Muthusamy Kunnimalaiyaan \\ University of Wisconsin School of Medicine and Public Health, Endocrine Surgery Research Laboratories, \\ Department of Surgery and University of Wisconsin Carbone Cancer Center, University of Wisconsin, \\ Madison, Wisc., USA
}

\section{Key Words}

Carcinoid $\cdot$ Pasireotide $\cdot$ Somatostatin analog $\cdot$

Teriflunomide $\cdot$ Neuroendocrine $\cdot$ Synergy

\begin{abstract}
Background/Aim: Somatostatin (SST) analogs are mainstay for controlling tumor proliferation and hormone secretion in carcinoid patients. Recent data suggest that extracellular signal-regulated kinase 1/2 (ERK1/2) phosphorylation may potentiate the anti-tumor effects of SST analogs in carcinoids. Additionally, ERK1/2 phosphorylating agents have been shown to suppress biomarker expression in carcinoids. Thus, Raf-1/MEK/ERK1/2 pathway activating drugs may be synergistic with SST analogs such as pasireotide (SOM230), which may be more effective than others in its class given its elevated receptor affinity and broader binding spectrum. Here, we investigate the effects of SOM230 in combination with teriflunomide (TFN), a Raf-1 activator, in a human carcinoid cell line. Methods: Human pancreatic carcinoid cells (BON) were incubated in TFN, SOM230 or a combination. Cell proliferation was measured using a rapid colorimetric assay. Western analysis was performed to analyze expression levels of achaete-scute complex-like 1 (ASCL1), chromogranin A (CgA), phosphorylated and total ERK1/2, and markers for apoptosis. Results: Combination treatment with SOM230
\end{abstract}

and TFN reduced cell growth beyond the additive effect of either drug alone. Combination indices $(\mathrm{Cl})$ fell below 1, thus quantifiably verifying synergy between both drugs as per the Chou-Talalay $\mathrm{Cl}$ scale. Combined treatment also reduced ASCL1 and CgA expression beyond the additive effect of either drug alone. Furthermore, it increased levels of phosphorylated ERK1/2, cleaved poly(ADP)-ribose polymerase and caspase-3, and reduced levels of anti-apoptotic biomarkers. Elevated phosphorylated ERK1/2 expression following combination therapy may underlie the synergistic interaction between the two drugs. Conclusion: Since efficacy is achieved at lower doses, combination therapy may palliate symptoms at low toxicity levels. Because each drug has already been evaluated in clinical trials, combinatorial drug trials are warranted.

Copyright $\odot 2012$ S. Karger AG, Basel

\section{Introduction}

Gastrointestinal (GI) and pancreatic carcinoids comprise a rare subtype of neuroendocrine tumors (NETs), representing nearly $2 \%$ of all malignant GI tumors, and reporting an incidence of $1-8$ per every 100,000 individuals $[1,2]$. Two thirds of carcinoids originate from the diffuse enterochromaffin cells of the gastrointestinal

\begin{tabular}{ll}
\hline KARGER & ( 2012 S. Karger AG, Basel \\
0028-3835/13/0972-0183\$38.00/0 \\
$\begin{array}{l}\text { Fax +41 61 306 } 1234 \\
\begin{array}{l}\text { E-Mail karger@karger.com } \\
\text { www.karger.com }\end{array}\end{array}$ & $\begin{array}{l}\text { Accessible online at: } \\
\text { www.karger.com/nen }\end{array}$
\end{tabular}

Muthusamy Kunnimalaiyaan

5131 Wisconsin Institutes for Medical Research

1111 Highland Ave.

Madison, WI 53705 (USA)

Tel. +1 608262 7441, E-Mail kunni@ surgery.wisc.edu 
tract and are typically classified according to their anatomical origin, arising from either the small intestine (41.8\%), rectum (27.4\%), or stomach (8.7\%) [3]. Pancreatic carcinoids most commonly occur sporadically (90\%), while the remainder of cases arise in the presence of hereditary syndromes [4]. Because they generally follow an indolent clinical course, carcinoids were originally thought to be benign. Despite this, carcinoids present daunting challenges in clinical management [5]. They are known for their propensity to hypersecrete an array of bioactive hormones, many of which are implicated in the carcinoid syndrome. Among these hormones are serotonin, growth hormone, and gastrin $[3,6]$. Carcinoids also express the basic helix-loop-helix transcription factor, achaete-scute complex-likel (ASCL1) and secrete the acidic glycopeptide known as chromogranin $\mathrm{A}(\mathrm{CgA})$, both reliable predictors of the malignant carcinoid syndrome and a poor prognosis [7-12]. Furthermore, carcinoids frequently result in isolated liver metastases, only second to colorectal carcinoma [13]. While cure for carcinoid patients with advanced liver metastases is achievable through early intervention, diagnosis is often delayed and incidental [14]. This underscores the need for novel therapeutic strategies to reduce tumor burden and control symptoms in carcinoid patients.

In lieu of surgery, targeted therapies have been a commonly chosen alternative for palliating symptoms and stabilizing disease in carcinoid patients. Following its identification as a pan inhibitory agent, the hormone somatostatin (SST) has been widely explored as a potential pharmacotherapeutic agent [14]. Today, long-acting SST analogs stand as the mainstay of long-term medical therapy for carcinoid patients. Recent PROMID studies have demonstrated the ability of SST analogs to stabilize tumor growth in metastatic midgut carcinoid tumors [15]. The clinical utility of SST analogs is primarily focused on symptomatic alleviation and improvement of quality of life, particularly as a subcutaneous or intramuscular depot formulation [16]. The SST hormone exerts its effects by binding to a panel of five SST receptor subtypes $\left(\mathrm{sst}_{1-5}\right)$, together comprising a family of G-protein-coupled receptors. Activation suppresses the secretion of a plethora of bioactive hormones and factors involved in cell growth and angiogenesis [17]. Therefore, SST analogs have been developed to target the same SST receptor panel and mimic the physiological effects of the native peptide on NET tissues.

Octreotide and lanreotide belong to the group of the most thoroughly researched SST analogs, and are both widely exploited for their strong binding affinity to the sstr profile in carcinoids [18]. Following phase III clinical trials, both lanreotide and octreotide were demonstrated to significantly ameliorate symptoms associated with the NETs while achieving minimal side effects [19]. While effective in inhibiting the secretion of bioactive products, octreotide along with many other synthetic derivatives of SST seem to have little or no activity as antiproliferative agents in vivo $[16,20]$. Overall, SST analogs each tend to display variable binding affinity to each of the five receptors, thus limiting their action only to lesions that possess a similar sstr receptor profile [21].

The recent identification of the novel SST analog pasireotide (SOM230) introduced a promising candidate for enhancing palliative success in metastatic carcinoid patients, especially to those refractory to the classic analogs octreotide and lanreotide. Compared to its predecessor octreotide, SOM230 possesses a binding affinity that is 30,5 and 40 times higher to $s_{1} t_{1}, s s t_{3}$, and $s t_{5}$, respectively $[15,18]$. Furthermore, studies have shown that metastatic carcinoid patients who did not respond to octreotide LAR experienced a reduction in symptoms of flushing and diarrhea when given SOM230 while still maintaining a similar safety profile to that of octreotide [22]. In vitro studies on bronchial carcinoids have demonstrated that SOM230 was at least as potent as octreotide, however, lacking statistically significant differences in the levels of hormone suppression [23]. Furthermore, there exists a paucity of in vitro data on the effects of SOM230 on expression of other neuroendocrine biomarkers or on cell proliferation in carcinoids.

Like most NETs, carcinoids grow as a result of modulating various downstream signaling pathways [13]. Recently, we have identified that induction of the Raf- $1 / \mathrm{mi}$ togen-activated protein kinase kinase (MEK)/extracellular signal-regulated kinase 1/2 (ERK1/2) pathway inhibits carcinoid biomarker expression. Raf-1/MEK/ERK1/2 pathway activation involves phosphorylation of Raf-1, a cytosolic serine/threonine kinase [24]. An activated Raf-1 phosphorylates MEK1/2 which subsequently phosphorylates ERK1/2 [6]. Our findings suggest that the Raf-1 pathway may serve as a viable target of activation in the treatment of carcinoid disease $[6,24,25]$. In human pancreatic carcinoid (BON) cell lines, the Raf-1 activator teriflunomide (TFN) was shown to suppress expression levels of ASCL1 and CgA, while pretreatment with the MEK1/2 inhibitor U0126 annulled these effects [6]. Thus, TFN's ability to suppress neuroendocrine biomarker expression in carcinoids was dependent on the presence of phospho-ERK1/2. We have also shown TFN to dose de- 
pendently inhibit carcinoid tumor proliferation both in vitro and in vivo [6].

In addition to its role in the regulation of carcinoid cell proliferation the Raf-1/MEK/ERK1/2 pathway has also been implicated in SST activity [26-29]. Furthermore, data from multiple NET cell lines demonstrated that treatment with SST analogs induce variable effects on tumor growth and ERK1/2 activation with respect to the unique SST receptor expression profile of each tissue examined in the panel [19]. Notably, it was reported that SST analog-induced growth inhibition occurred only in the presence of ERK1/2 phosphorylation, JNK signaling and activation of cdk inhibitors. This may suggest that SST analogs exert their antiproliferative effect through the Raf-1/MEK/ERK1/2 pathway [19]. Therefore, activating this pathway in conjunction with certain SST analogs would presumably enable or further potentiate their antitumorigenicity. Moreover, because Raf- 1 activation alone induces anti-tumor effects in carcinoids, Raf- 1 activators such as TFN may potentiate the effects of SST analogs when both are therapeutically administered in combination.

Here, for the first time, we demonstrate that combinatorial treatment with the novel multi-receptor SST analog SOM230 and TFN in carcinoid cell lines leads to synergistic inhibition of tumor cell proliferation and neuroendocrine biomarker levels while simultaneously increasing levels of phosphorylated ERK1/2. Furthermore, we demonstrate an apoptotic mechanism of growth inhibition that is induced following synergistic interplay between the two agents.

\section{Materials and Methods}

\section{Cell Culture and Treatment}

BON cells were provided by Drs. B. Mark Evers and Courtney M. Townsend, Jr. (University of Texas Medical Branch, Galveston, Tex., USA). BON cells were maintained in DMEM/F-12 (Life Technologies, Grand Island, N.Y., USA), supplemented with 10\% fetal bovine serum (Sigma-Aldrich, St. Louis, Mo., USA), 100 IU/ $\mathrm{ml}$ penicillin, and $100 \mu \mathrm{g} / \mathrm{ml}$ streptomycin (Life Technologies, Grand Island, N.Y., USA) in a humidified atmosphere of $5 \% \mathrm{CO}_{2}$ at $37^{\circ} \mathrm{C}$.

SOM230, graciously provided by Novartis ${ }^{\circledR}$, was dissolved in sterile double-distilled water at $10 \mathrm{~mm}$. TFN (Calbiochem ${ }^{\circledR}$, Darmstadt, Germany), was dissolved in dimethyl sulfoxide and stored at $100 \mathrm{mM}$. Both stock solutions were stored at $-80^{\circ} \mathrm{C}$. Drugs were diluted down in DMEM/F-12 to experimental concentrations, and prepared fresh for every experiment.

Cells were plated and allowed to adhere overnight. Cells were then treated with SOM230 alone, TFN alone, or a combination. SOM230 and TFN doses ranged from 0 to $10 \mu \mathrm{M}$, and 0 to $125 \mu \mathrm{M}$, respectively. Cells receiving a combination of the two drugs were first pretreated with TFN at either 35 or $50 \mu \mathrm{M}$ for $2 \mathrm{~h}$, after which SOM230 was added to the existing media, at concentrations ranging from 0 to $10 \mu \mathrm{M}$. All treatments were replenished every $48 \mathrm{~h}$. The total time of drug incubation was $96 \mathrm{~h}$.

\section{Cell Proliferation Assay}

Carcinoid tumor cell proliferation was measured using the 3-(4,5-dimethylthiazol-2-yl)-2,5-diphenyltetrazolium bromide (MTT) rapid colorimetric assay [30]. BON cells were plated in 24well plates and allowed to adhere overnight. Cells were then treated with SOM230 alone, TFN alone, or a combination, each in quadruplicate. SOM230 and TFN doses ranged from 0 to $10 \mu \mathrm{M}$ and 0 to $125 \mu \mathrm{M}$, respectively. Treatments lasted for up to $96 \mathrm{~h}$, and were replenished every $48 \mathrm{~h}$.

The MTT assay was performed by replacing the medium with $250 \mu \mathrm{l}$ of serum-free medium containing $0.5 \mathrm{mg} / \mathrm{ml}$ MTT. These were then incubated at $37^{\circ} \mathrm{C}$ for $3.5 \mathrm{~h}$. After incubation, $750 \mu \mathrm{l}$ of dimethyl sulfoxide (Fisher Scientific, Pittsburgh, Pa., USA) were added to each well and mixed thoroughly. The optical densities of each well were measured at $540 \mathrm{~nm}$ using a spectrophotometer ( $\mu$ Quant, Bio-Tek Instruments, Winooski, Vt., USA).

\section{Cell Viability Count Assay}

A trypan blue exclusion assay was performed to assess the effect of TFN on carcinoid cell growth and viability. Carcinoid tumor cells were counted and plated onto 6-well plates with approximately 100,000 cells and allowed to adhere overnight, after which cells were treated with select doses of TFN in triplicate for 4 days. Next, cells were collected following trypsinization, centrifuged, and resuspended in $300 \mu \mathrm{l}$ of PBS. Equal volumes of trypan blue and the cell suspension were then combined (10 $\mu$ l each), to create a 1:1 mixture, with which the number of cells (viable-unstained; non-viable-blue) was counted using a TC10 automated cell counter (Bio-Rad Laboratories Hercules, Calif., USA). These counts were normalized and subsequently compared with normalized MTT measurements for identically treated cells in parallel.

\section{Immunoblot Analysis}

Following treatment, cells were washed in ice-cold $1 \times$ PBS, and cellular pellets were lysed in sample buffer $(50 \mathrm{mM}$ Tris, $0.15 \mathrm{M} \mathrm{NaCl}, 0.5 \% \mathrm{Na}$ /deoxycholate, $0.1 \%$ SDS, $1 \%$ Nonidet P-40, and $0.6 \mathrm{~mm}$ phenylmethanesulfonylfluoride or phenylmethylsulfonyl fluoride) [13]. Total cellular protein concentrations were determined using the bicinchoninic acid assay (Pierce, Rockford, Ill., USA). Samples were then denatured and resolved on 7, 10, or $12 \%$ SDS-PAGE gels (Invitrogen, Grand Island, N.Y., USA), and transferred onto nitrocellulose membranes (Bio-Rad Laboratories, Hercules, Calif., USA). Protein-bound membranes were blocked for at least $30 \mathrm{~min}$ in a milk solution (1× PBS, 5\% dry milk, $0.05 \%$ Tween-20), and then incubated in respective primary antibodies overnight at $4^{\circ} \mathrm{C}$. Each antibody was diluted as follows: 1:2,000 for mammalian ASCL1 (BD PharMingen, San Diego, Calif., USA), 1:3,000 for CgA (Zymed Laboratories, San Francisco, Calif., USA), and 1:10,000 for glyceraldehyde-3-phosphate dehydrogenase (GAPDH; Trevigen, Gaithersburg, Md., USA), and 1:1,000 for phosphorylated ERK1/2 ${ }^{\text {thr202/tyr204 }}$, total ERK1/2, cleaved caspase-3, total caspase-3, cleaved poly(ADP)-ribose polymerase (PARP), X-linked inhibitor of apoptosis (XIAP), survivin, Mcl-1, and $\beta$-actin (Cell Signaling Technology, Beverly, 

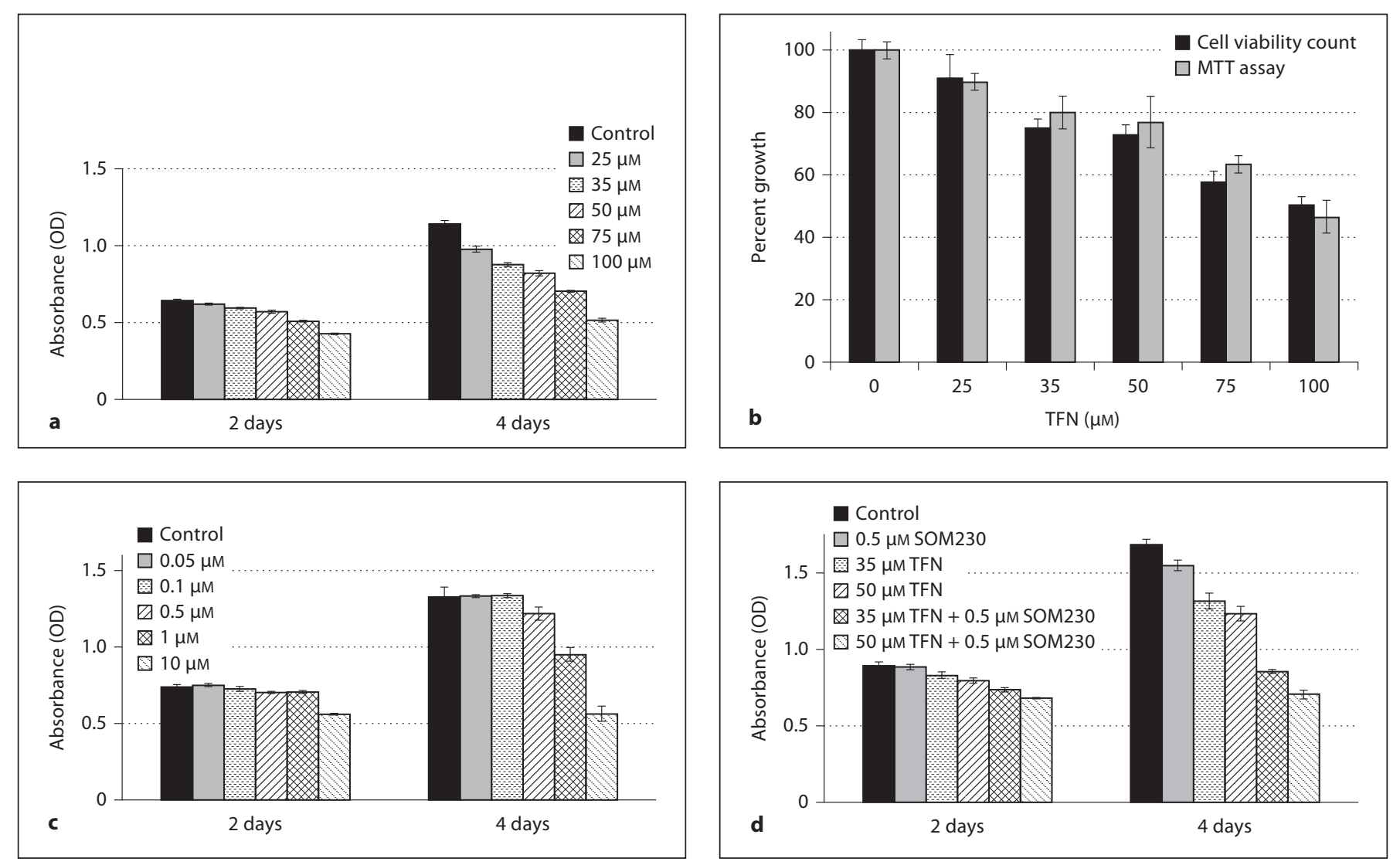

Fig. 1. Effect of TFN alone, SOM 230 alone and combination treatment on BON cell proliferation. a TFN dosedependently decreased BON cell growth after 4 days, with an $\mathrm{IC}_{50}$ of $96.2 \mu \mathrm{M}$. b Trypan blue exclusion assay confirmed the degree of growth inhibition measured by the MTT assay. c SOM230 inhibited growth at upper range doses, with an $\mathrm{IC}_{50}$ of $5.87 \mu \mathrm{M}$. d Combination treatment synergistically inhibited cell growth. CIs were calculated using the CompuSyn ${ }^{\circledR}$ software.

Mass., USA). Following incubation in primary antibody, membranes were washed $3 \times 5$ in PBS-T wash buffer $(1 \times$ phosphate buffered saline, $0.05 \%$ Tween 20 ), and subsequently incubated in horseradish peroxidase-conjugated goat anti-rabbit or antimouse secondary antibodies (Pierce, Rockford, Ill., USA) depending on the source of the primary antibody. Membranes were then washed $3 \times 5$ or $3 \times 10$ in PBS-T wash buffer and developed using Immunstar (Bio-Rad Laboratories, Hercules, Calif., USA), Supersignal West Pico or Femto (Pierce) kits according to the manufacturer's instructions.

\section{Statistical Analysis}

Quantity One software v. 4.6.3 (Bio-Rad Laboratories) was used to quantify density of Western blotting results. Using the CompuSyn ${ }^{\circledR}$ (Paramus, N.J., USA) software, combination index (CI) values were calculated for cells receiving combination therapy according to the Chou and Talalay mathematical model for drug interactions. A CI of more than, equal to, or less than 1 indicates antagonism, additivity, or synergy, respectively [31, 32]. All data are presented as mean \pm SEM, unless specifically noted.

\section{Results}

\section{Combined SOM230 and TFN Treatment}

Synergistically Inhibits Carcinoid Cell Proliferation

As we have reported, the Raf- 1 activator TFN inhibits cell proliferation and the expression of ASCL1 and CgA in carcinoid cells in vitro. We initially sought to determine TFN's anti-tumor effects by treating BON cells with increasing doses of TFN up to $100 \mu \mathrm{M}$ and establishing its $\mathrm{IC}_{50}$. Cell survival fractions were then determined 2 and 4 days following treatment using the MTT assay. Indeed, a dose-dependent trend in growth inhibition was observed accordingly, with an $\mathrm{IC}_{50}$ of $96.2 \mu \mathrm{M}$ of TFN following 4 days of treatment. TFN doses of 35 and $50 \mu \mathrm{M}$ resulted in cell survival of approximately 78 and $73 \%$, respectively (fig. 1a). To further confirm the pattern of cell growth inhibition, we carried out a viable cell count using 


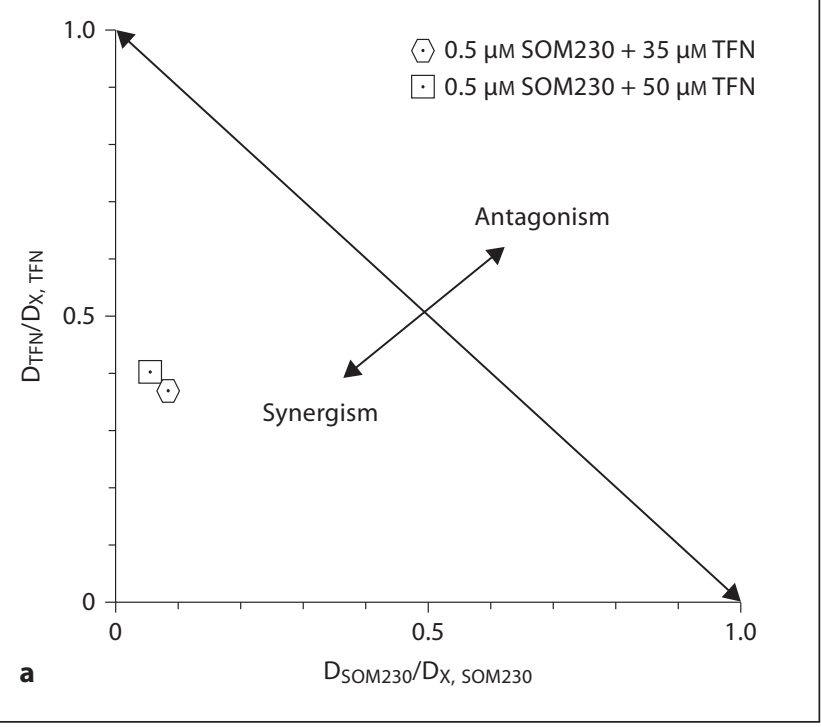

a trypan blue exclusion assay. Viability counts of BON cells treated with identical TFN doses also demonstrated a similar trend in growth inhibition, with doses of 35 and $50 \mu \mathrm{M}$ inhibiting cell proliferation by approximately 75 and $73 \%$, respectively (fig. 1 b). The dose range for SOM 230 treatment was determined based on past reported SOM230 protocols for the treatment of other neuroendocrine cell lines in vitro $[17,33]$. A range from 0 to $10 \mu \mathrm{M}$ of SOM230 was used to treat BON cells, and cell viability fractions were calculated using the MTT assay both 2 and 4 days following treatment. Data revealed that SOM230 only affected growth of BON cells at $0.5,1$ and $10 \mu \mathrm{M}$, with an $\mathrm{IC}_{50}$ of $5.87 \mu \mathrm{M}$ following 4 days of treatment, but had no significant effect below this range (fig. 1c).

Next, we wanted to determine the effect of combined low-dose TFN and SOM230 on cell proliferation. Because SOM230 treatment alone marginally inhibited cell growth at $0.5 \mu \mathrm{M}$ (by $8.2 \%$ ), we selected this concentration as the fixed low dose for combination treatment. By reducing the dose of TFN in combination with low-dose SOM230, we sought to demonstrate the potential for synergy to occur at low toxicity levels. When administered individually, TFN and SOM 230 each produced predictable effects on cell proliferation. Treatment with 35 and $50 \mu \mathrm{M}$ of TFN alone inhibited growth by approximately 22 and $27 \%$, respectively, while $0.5 \mu \mathrm{M}$ of SOM230 alone had marginal effect. When $0.5 \mu \mathrm{M}$ SOM230 was administered with either 35 or $50 \mu \mathrm{M}$ of TFN, cells exhibited growth inhibition of about 50 and 58\% respectively. Therefore, the anti-proliferative effect of SOM230, which

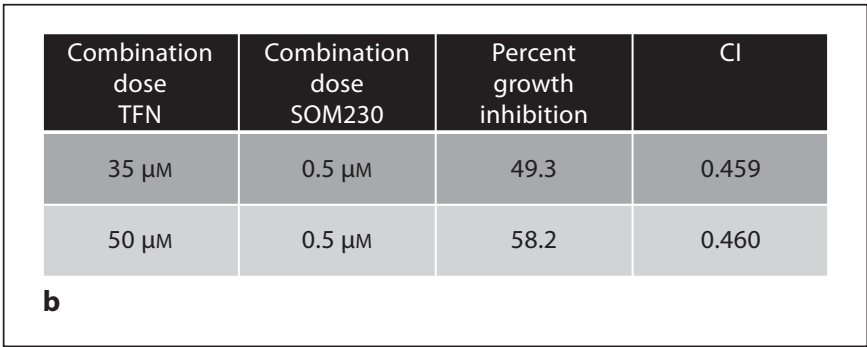

Fig. 2. CIs for experimental combination doses of TFN and SOM230 indicate synergistic growth inhibition of BON carcinoid cells. a Isobologram analysis of both combination treatment ratios that fall below the synergistic threshold. b CIs extrapolated from isobol analysis are $<1$, altogether strongly indicating a synergistic mode of interaction between SOM230 and TFN in BON carcinoid cells.

alone was inefficacious, was demonstrably enhanced by concomitant treatment with increasing doses of TFN (fig. 1d). The level of synergy at these dose combinations was further validated by isobol analysis and CIs (fig. 2), which were calculated for both experimental combination treatment regimens using the Chou-Talalay model for drug interaction. The CI values for both treatment combinations were well below 1 , indicating significant synergism between the drugs.

\section{TFN Potentiates the Effects of SOM230 on}

Neuroendocrine Biomarker Expression

We next sought to determine if TFN treatment would potentiate SOM230 to reduce neuroendocrine biomarker expression. Protein expression levels for both ASCL1 and CgA were assessed following combinatorial treatment with the two agents at select doses. We chose singly administered doses of SOM230 beginning at $0.5 \mu \mathrm{M}$ and ending at $10 \mu \mathrm{M}$, as this range inhibited cell growth in proliferation studies by 8.43 up to $57.68 \%$, respectively (fig. 1c). A fixed TFN dose of $50 \mu \mathrm{M}$ was chosen for combination since this concentration inhibited cell proliferation by $28.0 \%$, thus allowing for a sufficient window of synergy to be observed at the level of protein expression. Results indicated that the addition of increasing doses of SOM230 to pretreated, Raf-1-activated BON cells resulted in a progressive reduction in the expression of $\mathrm{CgA}$ (fig. 3a). While $50 \mu \mathrm{M}$ of TFN alone inhibited CgA expression levels to $47 \%$ of baseline levels, combined treatment with SOM230 progressively reduced expression lev- 

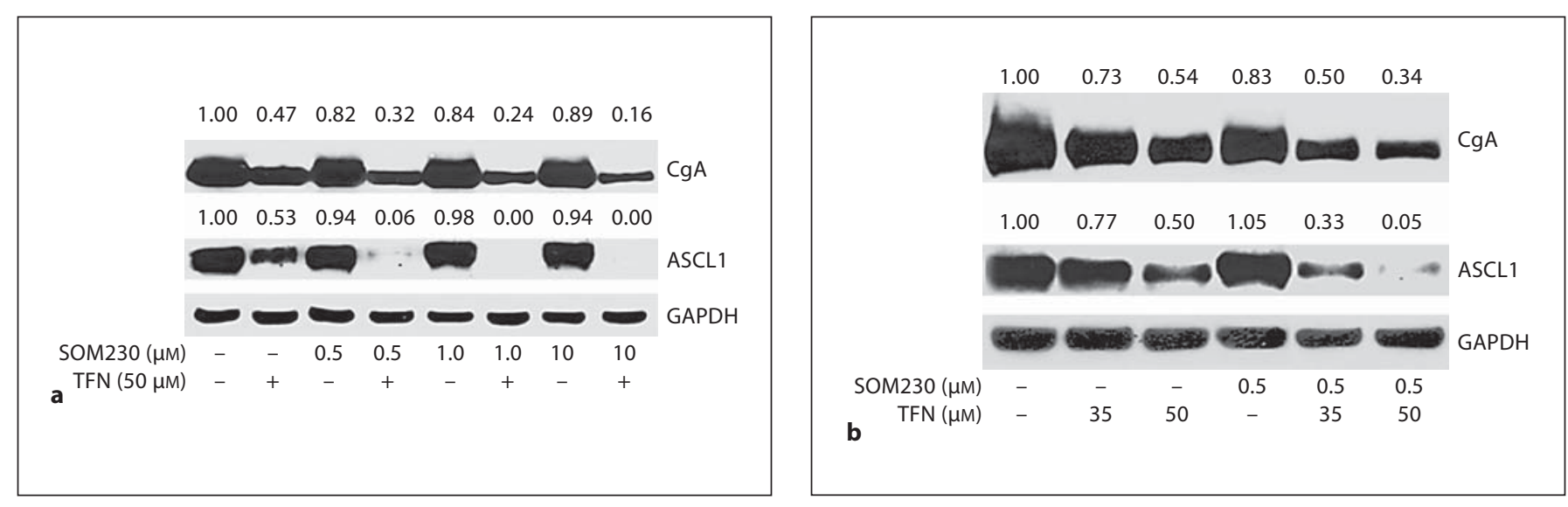

Fig. 3. Combination therapy synergistically inhibits expression of CgA and ASCL1 in BON cells. a While increasing doses of SOM230 alone only minimally affected CgA and ASCL1 expression levels, concomitant treatment with $50 \mu \mathrm{M}$ of TFN potentiated SOM230 to cause up to 84 and 100\% inhibition of CgA and ASCL1, respectively. $\mathbf{b}$ The low-dose combinations of TFN and SOM230 were shown to inhibit CgA and ASCL1 expression beyond the additive effect of either drug dosage alone. Both $\mathbf{a}$ and $\mathbf{b}$ present densitometric values as normalized to their respective GAPDH loading control and subsequently to their respective control densities.

els to 32,24 , and $16 \%$ following SOM230 doses of 0.5 , 1 and $10 \mu \mathrm{M}$, respectively. Notably, combined treatment with the two agents nearly depleted levels of ASCL1 expression at SOM230 doses higher than $1 \mu \mathrm{M}$ in combination with $50 \mu \mathrm{M}$ TFN, and left only $6 \%$ of baseline ASCL1 expression with $0.5 \mu \mathrm{M}$ SOM230 together with $50 \mu \mathrm{M}$ TFN. In contrast, $50 \mu \mathrm{M}$ of TFN alone suppressed ASCL1 expression to only $53 \%$ of baseline. Treatment with SOM230 alone within this dose range produced no significant effect on the expression of these biomarkers, as expected.

Having demonstrated that TFN potentiates a range of SOM230 doses from 0.5 to $10 \mu \mathrm{M}$, our next aim was to determine if lower doses of both TFN and SOM230 could synergistically inhibit ASCL1 and CgA expression when administered in combination. Western analysis did indeed reveal that pretreatment with TFN doses at $35 \mu \mathrm{M}$ were sufficient to potentiate $0.5 \mu \mathrm{M}$ SOM230 to inhibit ASCL1 and CgA expression (fig. 3b). Data showed that while $35 \mu \mathrm{M}$ of TFN alone effectively reduced CgA and ASCL1 levels to 73 and $77 \%$, respectively, subsequent treatment with $0.5 \mu \mathrm{M}$ of SOM 230 reduced $\mathrm{CgA}$ and ASCL1 expression levels to 50 and $33 \%$, respectively. This dose of SOM230 alone, however, had no effect on biomarker expression. Increasing the pretreatment dosage of TFN to $50 \mu \mathrm{M}$ before administering $0.5 \mu \mathrm{M}$ of SOM 230 further reduced CgA and ASCL1 levels to 34 and 5\%, respectively. In contrast, cells receiving only $50 \mu \mathrm{M}$ of
TFN expressed ASCL1 and CgA levels at about 50\% of baseline levels. Thus, increasing the dose of TFN enhanced the sensitivity of the BON carcinoid cell line to low-dose SOM230. Importantly, the level of neuroendocrine biomarker inhibition following combination treatment was markedly greater than the additive effect of either SOM230 or TFN alone, particularly because $0.5 \mu \mathrm{M}$ of SOM230 was by itself ineffective. These data suggest that low doses of TFN sensitize BON carcinoid cells to lowdose SOM230, and do so in a dose-dependent manner.

\section{Combined TFN and SOM230 Treatment Increases}

the Phosphorylation of ERK1/2 in vitro

Next, we sought to investigate the activity of the Raf-1 pathway following combination treatment with low-dose SOM230 and TFN. Cells receiving $0.5 \mu \mathrm{M}$ of SOM 230 in combination with 35 or $50 \mu \mathrm{M}$ of TFN expressed significantly higher levels of phosphorylated ERK1/2 compared to cells receiving the same doses of TFN or SOM230 individually (fig. 4a). While TFN, known for its Raf-1 activation-dependent anti-tumor effects, expectedly increased levels of phospho-ERK1/2, it did so to a significantly lesser extent than that achieved with combination therapy. SOM230 alone had minimal effects on phosphoERK1/2 expression, altogether suggesting that TFN potentiates SOM230 to induce phospho-ERK1/2 expression beyond the additive effect of either drug alone. Additionally, this demonstrates a correlation between ERK1/2 

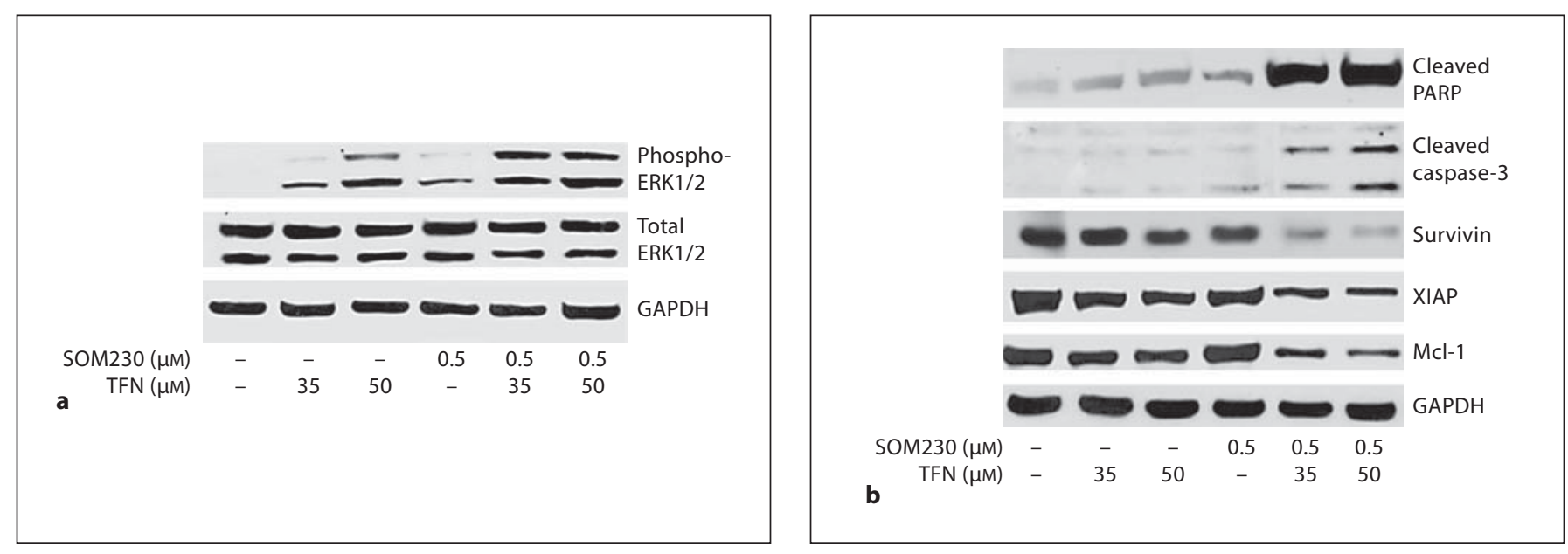

Fig. 4. Combination therapy activates the Raf-1/MEK/ERK pathway and promotes apoptosis. a While SOM230 alone only marginally activated the ERK1/2 pathway, combination treatment with TFN increased phospho-ERK1/2 expression levels beyond the additive effect of either drug alone. $\mathbf{b}$ Cleavage of PARP and caspase-3 and the inhibition of anti-apoptotic molecular markers following combination therapy demonstrate the induction of apoptosis.

phosphorylation and the synergistic inhibition of neuroendocrine biomarker expression observed following combination treatment.

\section{Combined TFN and SOM230 Treatment Induces \\ Apoptosis}

TFN's anti-cancer properties have been well attributed to the induction of cell cycle arrest and apoptosis $[6,34$, $35]$. In order to determine the mechanism of growth inhibition induced by the interplay between TFN and SOM230 in BON carcinoid cells, we performed Western blot analysis for markers of apoptosis and cell cycle progression (fig. 4b). BON carcinoid cells were treated with the same concentrations of SOM 230 and TFN, both individually and in combination as described above. Combination treatment led to significantly elevated levels of cleaved PARP and cleaved caspase- 3 compared to the effect produced by either drug alone. When administered individually, both TFN and SOM230 only slightly increased levels of cleaved PARP and caspase-3, though markedly less than combined treatment. However, lowdose SOM230 treatment resulted in increased levels of PARP and caspase- 3 cleavage when administered with 35 and $50 \mu \mathrm{M}$ of TFN. Because PARP and caspase- 3 cleavage are both definitive molecular indicators of apoptosis, these findings strongly suggest an apoptotic mechanism of synergy following the TFN and SOM230 combination treatment. Upon further investigation, we demonstrated a decrease in the expression of various anti-apoptotic factors following combination treatment (fig. 4b). This further validated the induction of apoptosis following combination treatment with TFN and SOM230. Low-dose treatment with the two agents markedly reduced levels of anti-apoptotic markers, including survivin, XIAP, and Mcl-1, beyond the effect achieved by either drug alone. While TFN administered alone dose-dependently reduced the expression of these molecular markers, it did so only slightly. SOM230 once again did not show any effect on pro-apoptotic biomarker expression. Consequently, the inhibitory effect achieved following combination therapy exceeded the cumulative effect achieved by either drug. Finally, higher doses of TFN managed to further reduce levels of these anti-apoptotic markers when administered in conjunction with the fixed low dose of SOM230, underscoring the dose dependency of sensitization to SOM230 with increasing TFN treatment.

\section{Discussion}

Due to the high frequency of hepatic metastases and the carcinoid syndrome, carcinoid tumors are often unresectable at the time of diagnosis, and can be difficult to manage medically $[6,13,36,37]$. Recent studies maintain that certain adjuvant therapies such as SST analogs lanreotide and octreotide typically diminish in effect follow- 
ing prolonged therapy, and lack much efficacy in carcinoid patients $[6,14]$. In cases where patients develop resistance to SST analog treatment, achieving the desired therapeutic effect may require higher doses, leading to higher toxicitiy and adverse side effects. This has demanded the development of low-dose therapeutic alternatives.

The identification of pasireotide (SOM230) as a novel SST analog therapy introduced a promising therapeutic candidate for carcinoid patients. SOM230 has a higher functional activity for sstr5 than octreotide, and a longer half-life than either octreotide or lanreotide [21, 38]. Combined with a multi-receptor spectrum that resembles the endogenous SST hormone, SOM230 may exert magnified effects relative to sstr2-preferential analogs octreotide and lanreotide [14]. Phase II randomized clinical trials on acromegaly patients have demonstrated that SOM230 achieves a significantly increased therapeutic effect and longer duration of action compared to octreotide $[18,39]$.

Despite hopeful projections, SOM230 has been used with varied success in clinical trials, and its advantage over alternative SST analogs remains indeterminate [18, 39]. Similar trials on carcinoid patients have demonstrated improved manageability of carcinoid syndrome symptoms following SOM230 treatment, but its effect on tumor volume is just as inefficacious as octreotide's [21]. Our data demonstrate at the in vitro level that carcinoid BON cells exhibited minimal effects on the expression of ASCL1 and CgA following treatment with SOM230. These biomarkers are strong indicators of carcinoid malignancy, and so their expression levels can be used to assess the degree of drug efficacy against these tumors [611]. Thus, these data corroborate empirical evidence of SOM230's limited potential in controlling carcinoid tumor growth in only certain patients.

SOM230's differential cytotoxicity in carcinoids may be contingent on the activity of associated signaling pathways. We believe that SOM230's anti-tumor effects may be inexorably linked to the Raf-1/MEK/ERK1/2 cascade, as evidenced by certain SST analogs that exert their antitumor effects in the presence of high levels of phosphorylated ERK1/2 [19]. This may suggest why certain carcinoid tissues that exhibit low levels of endogenous Raf-1 pathway activation would fail to respond to SST analog therapies such as SOM230. Our group has shown that activation of the Raf-1/MEK/ERK1/2 pathway alone, both endogenously as well as following treatment with the Raf-1 activator TFN, can inhibit neuroendocrine biomarker expression and phenotypic differentiation among various NET subtypes, including pancreatic carcinoids $[6,11,40]$.
In this study, we demonstrate that SOM230's anti-carcinoid effects are potentiated following combinatorial treatment with TFN. We therefore propose that SOM230 in combination with TFN can be a novel and efficacious therapeutic option for carcinoid patients. Low-dose combination therapy with both of these agents inhibited cell proliferation as well as ASCL1 and CgA expression beyond the additive effect of either drug alone. The antitumor effect occurred through the induction of apoptosis, evidenced by an increased expression of pro-apoptotic markers and suppression of anti-apoptotic factors.

Traditionally, activation of the Raf- 1 pathway has been thought to confer tumorigenic properties as documented in multiple cancer types, including papillary thyroid carcinoma, non-small-cell lung cancer and hepatocellular carcinoma [41, 42]. Conversely, the Raf-1 pathway has been recognized for its anti-tumor effects in carcinoids as well as other NETs $[6,10,13,24,25,40,43,44]$. Only minimal baseline levels of phosphorylated ERK1/2 are observed in carcinoid cell lines. In the past, we have shown that activation of this pathway reduces the expression of ASCL1, CgA and serotonin not only in carcinoids, but other types of NETs such as medullary thyroid cancers and small cell lung cancers as well $[6,10,13,25,43-$ 45]. Interestingly, our findings in carcinoids demonstrate an increase in levels of phosphorylated ERK1/2 alongside synergistic growth inhibition following combined SOM230 and TFN treatment. This is well aligned with data of Kidd et al. [19], who suggest that the antiproliferative effect of SST analogs in NETs requires the presence of phosphorylated ERK1/2. This finding may point to a potential downstream mechanism by which these drugs interact. Because BON carcinoid cells exhibit low levels of phosphorylated ERK1/2 at baseline, this may in part explain their resistance to SOM230's antiproliferative effect. Therefore, TFN's potential for synergizing with SOM230 treatment in carcinoids may be attributed to its utility as an anti-tumor agent itself, in addition to its potential capacity to mobilize SOM230's anti-tumor properties via the activation of the Raf-1 pathway, in otherwise refractory tissue types. Based on the synergistic interaction between the downstream effects of SOM230 and TFN, combined therapy with these two agents may provide a novel strategy for enhancing tumor growth inhibition via the activation of a potent downstream anticarcinoid target.

The selection of TFN and SOM230 among alternative agents in their respective drug classes was determined based on their overall projected efficacy, accessibility, and clinical utility $[6,24,39,46]$. TFN along with its par- 
ent drug leflunomide (LFN), have both been approved by the Food and Drug Administration, and are known to be safe in humans [6]. LFN upon administration in humans is nearly completely converted to its active metabolite TFN through first pass metabolism [47, 48]. We have demonstrated that both TFN and LFN individually exert the same anti-cancer properties in carcinoid cell lines while activating the Raf-1 pathway. These drugs have already been used widely as immunomodulatory drugs, and so their pharmacokinetics, bioavailability, and therapeutic window are already well understood. Plasma concentrations of TFN in human rheumatoid arthritis patients receiving oral LFN doses of more than $200 \mu \mathrm{M}$ are comparable to the doses administered to carcinoid-bearing mouse models that responded to therapy $[6,49]$.

Given our findings, combination therapy with SOM230 and the Raf-1-activating agent TFN presents a promising option for reducing carcinoid tumor growth and syndrome symptomatology. Because a commensurate therapeutic effect can be achieved at lower doses of both drugs when administered simultaneously, com- pared to doses for either drug administered alone, combination therapy may be a valuable option for refractory patients or for those who respond poorly to higher toxicity levels at higher doses. We conclude that combination therapy with SOM 230 and TFN is worth further investigation in a phase II clinical trial.

\section{Acknowledgements}

This research was supported under the Howard Hughes Medical Institute Medical Fellowship Program (Y.S.), NIH grant R01 CA121115 (H.C.), the American Cancer Society Research Scholars Grant (H.C.), American Cancer Society MEN2 Professorship (H.C.), and NIH grant R03 CA155691 (M.K.). This research was also supported in part by grant P30 CA014520 from the National Cancer Institute. A special thanks to Novartis for supplying pasireotide.

\section{Disclosure Statement}

No potential conflicts of interest were disclosed.

\section{References}

1 Modlin I, Sandor A: An analysis of 8305 cases of carcinoid tumors. Cancer 1997;79:813829.

-2 Läuffer J, Zhang T, Modlin I: Review article: current status of gastrointestinal carcinoids. Aliment Pharmacol Ther 1999;13:271-287.

- 3 Maroun J, Kocha W, Kvols L, Bjarnason G, Chen E, Germond C, Hanna S, Poitras P, Rayson D, Reid R, Rivera J, Roy A, Shah A, Sideris L, Siu L, Wong R: Guidelines for the diagnosis and management of carcinoid tumours. 1. The gastrointestinal tract. A statement from a Canadian National Carcinoid Expert Group. Curr Oncol 2006;13:67-76.

4 Oberg K: Genetics and molecular pathology of neuroendocrine gastrointestinal and pancreatic tumors (gastroenteropancreatic neuroendocrine tumors). Curr Opin Endocrinol Diabetes Obes 2009;16:72-78.

5 Pasieka J: Carcinoid tumors. Surg Clin North Am 2009;89:1123-1137.

-6 Cook M, Pinchot S, Jaskula-Sztul R, Luo J, Kunnimalaiyaan M, Chen H: Identification of a novel Raf-1 pathway activator that inhibits gastrointestinal carcinoid cell growth. Mol Cancer Ther 2010;9:429-437.

7 Jiang S, Kameya T, Asamura H, Umezawa A, Sato Y, Shinada J, Kawakubo Y, Igarashi T, Nagai K, Okayasu I: hASH1 expression is closely correlated with endocrine phenotype and differentiation extent in pulmonary neuroendocrine tumors. Mod Pathol 2004; $17: 222-229$

Combination Treatment in Carcinoids
8 Seregni E, Ferrari L, Bajetta E, Martinetti A, Bombardieri E: Clinical significance of blood chromogranin A measurement in neuroendocrine tumours. Ann Oncol 2001; 12(suppl 2):S69-S72.

-9 Davis Z, Moertel C, Mcllrath D: The malignant carcinoid syndrome. Surg Gynecol Obstet 1973;137:637-644.

10 Kunnimalaiyaan M, Chen H: The Raf-1 pathway: a molecular target for treatment of select neuroendocrine tumors? Anticancer Drugs 2006; 17:139-142.

$\checkmark 11$ Chen H, Biel M, Borges M, Thiagalingam A, Nelkin B, Baylin S, Ball D: Tissue-specific expression of human achaete-scute homologue-1 in neuroendocrine tumors: transcriptional regulation by dual inhibitory regions. Cell Growth Differ 1997;8:677-686.

12 Tomassetti P, Migliori M, Simoni P, Casadei R, De Iasio R, Corinaldesi R, Gullo L: Diagnostic value of plasma chromogranin $\mathrm{A}$ in neuroendocrine tumours. Eur J Gastroenterol Hepatol 2001;13:55-58.

13 Sippel R, Carpenter J, Kunnimalaiyaan M, Lagerholm S, Chen H: Raf-1 activation suppresses neuroendocrine marker and hormone levels in human gastrointestinal carcinoid cells. Am J Physiol Gastrointest Liver Physiol 2003;285:G245-G254.

14 Modlin I, Pavel M, Kidd M, Gustafsson B: Review article: somatostatin analogues in the treatment of gastroenteropancreatic neuroendocrine (carcinoid) tumours. Aliment Pharmacol Ther 2010;31:169-188.
15 Culler MD, Oberg K, Arnold R, Krenning EP, Sevilla I, Diaz JA: Somatostatin analogs for the treatment of neuroendocrine tumors. Cancer Metastasis Rev 2011;30(suppl 1):917.

16 Modlin I, Latich I, Kidd M, Zikusoka M, Eick G: Therapeutic options for gastrointestinal carcinoids. Clin Gastroenterol Hepatol 2006;4:526-547.

17 Pasquali D, Rossi V, Conzo G, Pannone G, Bufo P, De Bellis A, Renzullo A, Bellastella G, Colao A, Vallone G, Bellastella A, Sinisi A: Effects of somatostatin analog SOM230 on cell proliferation, apoptosis, and catecholamine levels in cultured pheochromocytoma cells. J Mol Endocrinol 2008;40:263-271.

18 van der Hoek J, van der Lelij A, Feelders R, de Herder W, Uitterlinden P, Poon K, Boerlin V, Lewis I, Krahnke T, Hofland L, Lamberts S: The somatostatin analogue SOM230, compared with octreotide, induces differential effects in several metabolic pathways in acromegalic patients. Clin Endocrinol (Oxf) 2005;63:176-184.

19 Kidd M, Drozdov I, Joseph R, Pfragner R, Culler M, Modlin I: Differential cytotoxicity of novel somatostatin and dopamine chimeric compounds on bronchopulmonary and small intestinal neuroendocrine tumor cell lines. Cancer 2008;113:690-700.

20 Patel YC, Srikant CB: Somatostatin receptors. Trends Endocrinol Metab 1997;8:398405 . 
21 Ben-Shlomo A, Melmed S: Pasireotide - a somatostatin analog for the potential treatment of acromegaly, neuroendocrine tumors and Cushing's disease. IDrugs 2007; 10:885895.

22 Kvols L, Oberg K, Glusman JE, O’dorisio TM, De Herder W, Gao B, Arnold R, Anthony L, The SOM230 Carcinoid Study Group: Safety and efficacy of pasireotide (SOM230) in patients with metastatic carcinoid tumors refractory or resistant to octreotide LAR: results of a phase II study; in Am Soc Clin Oncol Annu Meet, Atlanta, June 2006.

-23 van Hoek M, Hofland L, de Rijke Y, van Nederveen F, de Krijger R, van Koetsveld P, Lamberts S, van der Lely A, de Herder W, Feelders R: Effects of somatostatin analogs on a growth hormone-releasing hormone secreting bronchial carcinoid, in vivo and in vitro studies. J Clin Endocrinol Metab 2009; 94:428-433.

-24 Van Gompel J, Kunnimalaiyaan M, Holen K, Chen H: ZM336372, a Raf-1 activator, suppresses growth and neuroendocrine hormone levels in carcinoid tumor cells. Mol Cancer Ther 2005;4:910-917.

25 Kappes A, Vaccaro A, Kunnimalaiyaan M, 36 Chen H: ZM336372, a Raf-1 activator, inhibits growth of pheochromocytoma cells. J Surg Res 2006;133:42-45.

-26 Florio T, Thellung S, Arena S, Corsaro A, Bajetto A, Schettini G, Stork P: Somatostatin receptor 1 (SSTR1)-mediated inhibition of cell proliferation correlates with the activation of the MAP kinase cascade: role of the phosphotyrosine phosphatase SHP-2. J Physiol Paris 2000;94:239-250.

-27 Schlessinger J, Ullrich A: Growth factor signaling by receptor tyrosine kinases. Neuron 1992;9:383-391.

28 Ullrich A, Schlessinger J: Signal transduction by receptors with tyrosine kinase activity. Cell 1990;61:203-212.

-29 Dhanasekaran N, Heasley L, Johnson G: G protein-coupled receptor systems involved in cell growth and oncogenesis. Endocr Rev 1995; 16:259-270.

- 30 Greenblatt D, Vaccaro A, Jaskula-Sztul R, Ning L, Haymart M, Kunnimalaiyaan $M$, Chen H: Valproic acid activates notch-1 signaling and regulates the neuroendocrine phenotype in carcinoid cancer cells. Oncologist 2007;12:942-951.
31 Zhao L, Wientjes M, Au J: Evaluation of combination chemotherapy: integration of nonlinear regression, curve shift, isobologram, and combination index analyses. Clin Cancer Res 2004;10:7994-8004.

32 Chou T, Talalay P: Quantitative analysis of dose-effect relationships: the combined effects of multiple drugs or enzyme inhibitors. Adv Enzyme Regul 1984;22:27-55.

33 Cescato R, Loesch K, Waser B, Mäcke H, Rivier J, Reubi J, Schonbrunn A: Agonist-biased signaling at the sst $2 \mathrm{~A}$ receptor: the multi-somatostatin analogs KE108 and SOM230 activate and antagonize distinct signaling pathways. Mol Endocrinol 2010;24:240-249.

34 Baumann P, Mandl-Weber S, Völkl A, Adam C, Bumeder I, Oduncu F, Schmidmaier R: Dihydroorotate dehydrogenase inhibitor A771726 (leflunomide) induces apoptosis and diminishes proliferation of multiple myeloma cells. Mol Cancer Ther 2009;8:366375.

35 Ringshausen I, Oelsner M, Bogner C, Peschel C, Decker T: The immunomodulatory drug Leflunomide inhibits cell cycle progression of B-CLL cells. Leukemia 2008;22:635-638.

6 Miller C, Ellison E: Therapeutic alternatives in metastatic neuroendocrine tumors. Surg Oncol Clin N Am 1998;7:863-879.

- 37 Chen H, Hardacre J, Uzar A, Cameron J, Choti M: Isolated liver metastases from neuroendocrine tumors: does resection prolong survival? J Am Coll Surg 1998;187:88-92, discussion 92-93.

38 Schmid H: Pasireotide (SOM230): development, mechanism of action and potential ap plications. Mol Cell Endocrinol 2008;286: 69-74.

39 Petersenn S, Schopohl J, Barkan A, Mohideen P, Colao A, Abs R, Buchelt A, Ho Y, Hu K, Farrall A, Melmed S, Biller B, Group PAS: Pasireotide (SOM230) demonstrates efficacy and safety in patients with acromegaly: a randomized, multicenter, phase II trial. J Clin Endocrinol Metab 2010;95:2781-2789.

40 Ravi R, Thiagalingam A, Weber E, McMahon M, Nelkin B, Mabry M: Raf-1 causes growth suppression and alteration of neuroendocrine markers in DMS53 human smallcell lung cancer cells. Am J Respir Cell Mol Biol 1999;20:543-549.

41 Gollob J, Wilhelm S, Carter C, Kelley S: Role of Raf kinase in cancer: therapeutic potential of targeting the Raf/MEK/ERK signal transduction pathway. Semin Oncol 2006;33:392406.
42 Davies H, Bignell G, Cox C, Stephens P, Edkins S, Clegg S, Teague J, Woffendin H, Garnett M, Bottomley W, Davis N, Dicks E, Ewing R, Floyd Y, Gray K, Hall S, Hawes R, Hughes J, Kosmidou V, Menzies A, Mould C, Parker A, Stevens C, Watt S, Hooper S, Wilson R, Jayatilake H, Gusterson B, Cooper C, Shipley J, Hargrave D, Pritchard-Jones K, Maitland N, Chenevix-Trench G, Riggins G, Bigner D, Palmieri G, Cossu A, Flanagan A, Nicholson A, Ho J, Leung S, Yuen S, Weber B, Seigler H, Darrow T, Paterson H, Marais R, Marshall C, Wooster R, Stratton M, Futreal P: Mutations of the BRAF gene in human cancer. Nature 2002;417:949-954.

43 Vaccaro A, Chen H, Kunnimalaiyaan M: In-vivo activation of Raf-1 inhibits tumor growth and development in a xenograft model of human medullary thyroid cancer. Anticancer Drugs 2006;17:849-853.

44 Chen H, Kunnimalaiyaan M, Van Gompel J: Medullary thyroid cancer: the functions of raf-1 and human achaete-scute homologue-1. Thyroid 2005; 15:511-521.

45 Sippel R, Carpenter J, Kunnimalaiyaan M, Chen H: The role of human achaete-scute homolog-1 in medullary thyroid cancer cells. Surgery 2003;134:866-871; discussion 871873.

46 Pinchot S, Adler J, Luo Y, Ju J, Li W, Shen B, Kunnimalaiyaan M, Chen H: Tautomycin suppresses growth and neuroendocrine hormone markers in carcinoid cells through activation of the Raf-1 pathway. Am J Surg 2009;197:313-319.

47 Tallantyre E, Evangelou N, Constantinescu C: Spotlight on teriflunomide. Int MS J 2008; 15:62-68.

48 Mladenovic V, Domljan Z, Rozman B, Jajic I, Mihajlovic D, Dordevic J, Popovic M, Dimitrijevic M, Zivkovic M, Campion G: Safety and effectiveness of leflunomide in the treatment of patients with active rheumatoid arthritis. Results of a randomized, placebocontrolled, phase II study. Arthritis Rheum 1995;38:1595-1603.

49 Chong A, Huang W, Liu W, Luo J, Shen J, Xu W, Ma L, Blinder L, Xiao F, Xu X, Clardy C, Foster P, Williams J: In vivo activity of leflunomide: pharmacokinetic analyses and mechanism of immunosuppression. Transplantation 1999;68:100-109. 